\title{
Thirty Years of Technology at Work for Health: Niurka Carlos, Director, Immunoassay Center, Havana
}

\section{Gail Reed MS}

Her passion for the "new world of informatics" led engineer Niurka Carlos to study computer sciences and digital systems, and her passion for health led her to join the embryo of Cuba's Immunoassay Center in 1982 right after graduation from university in 1987, becoming one of the Center's founders when it was launched that same year. First specializing in software, Ms Carlos was promoted to department chief, the Center's management team and later to deputy director. She has headed the Center since 2014, leading implementation of research, production and marketing of diagnostic tools for the national health system and export. Today, the Center is expanding its horizons into chronic diseases that have become the main causes of illness and death in Cuba and are afflicting low- and middle-income countries at alarming rates as part of the world's epidemiological transition. MEDICC Review spoke with Ms Carlos about the history, results, challenges and new frontiers of the Center in its $30^{\text {th }}$ anniversary year.

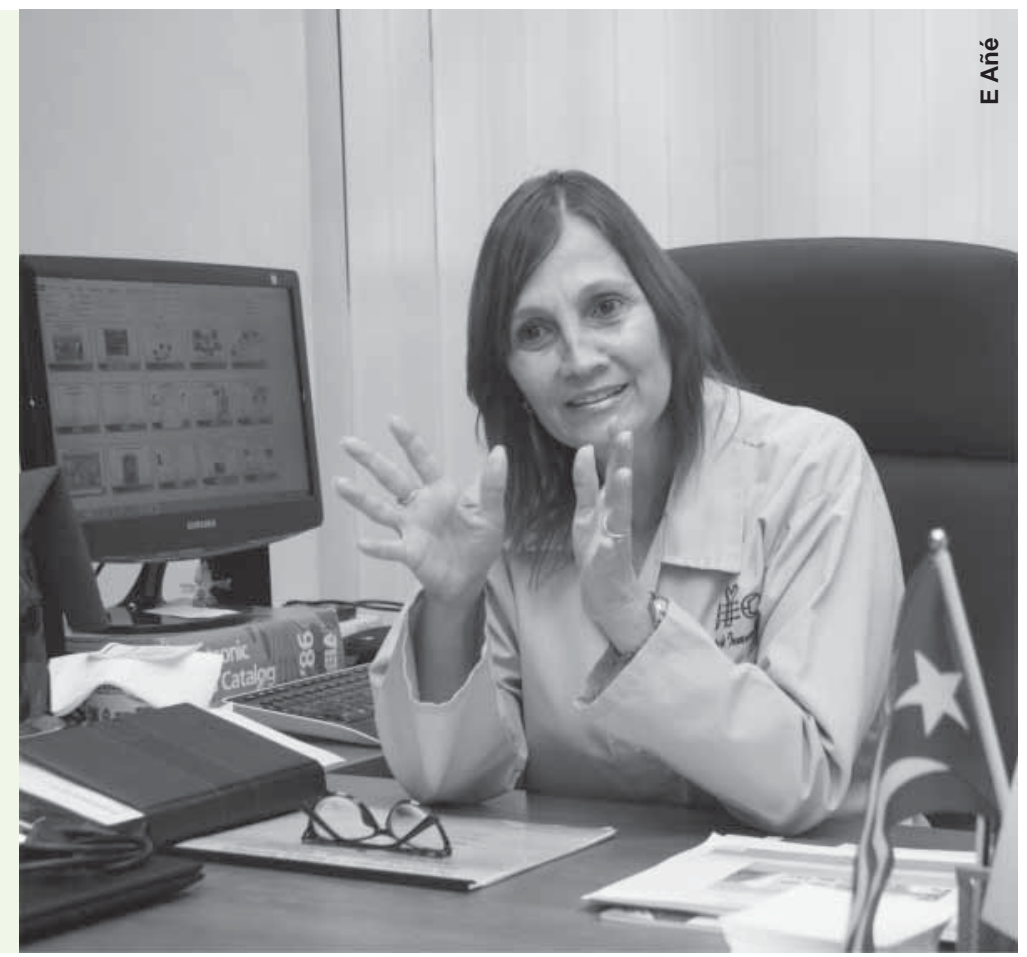

\section{MEDICC Review: How did the Immunoassay Center get its start?}

Niurka Carlos: Like many of our research centers, our group started working to address a health problem. In this case, it was how to carry out economically viable alpha-fetoprotein tests on pregnant women, to determine if they were carrying a fetus with neural tube defect-that is, a condition in which the neural tube doesn't close, and that can be incompatible with life. Our founding director, Dr José Luis Fernández Yero, was working with our team to develop our own ultra-microanalytic system technology (SUMA) that could be used by the public health system for widespread screening. We started with women in Havana, and in 1982, began introducing it throughout the country. This test, combined with genetic counseling, allows women and their partners to make informed decisions about whether or not to continue the pregnancy. Thus the technology contributes both to lower infant mortality and to better care for newborns with such a condition. Once was the test available throughout the health system, Cuba became the second country in the world to offer this test to all pregnant women.

From this first experience, the Center has engaged in research and development of diagnostic tools, instruments and software for maternal-child health (ante- and postnatal tests), as well as for infectious and most recently, chronic non-communicable diseases. Political will is key to our ability to introduce our technologies into national screening programs and patient care, political will in our case represented by the universal nature of Cuba's public health system.
I should mention here that the Center does not receive a budget from the central government: rather, we are one of the state companies in the BioCubaFarma business group. The concept is that we finance our R\&D, manufacture, marketing, training and technical assistance through our sales. We offer our products at very modest prices to the Cuban health system, and export internationally to a number of countries.

MEDICC Review: How does the Center provide its services, and what products do you provide to the national health system in Cuba and abroad?

Niurka Carlos: Our technologies cover a wide variety of uses, introduced since 1982. For example, we produce 26 diagnostic kits for 19 conditions, ranging from congenital malformations to HIVIAIDS, hepatitis B and C, diabetes and various cancers. All of these have been introduced nationwide in Cuba

In particular, we have achieved what we call "technology sovereignty," producing diagnostics, instruments and software for eight national screening and patient care programs: maternal-child health; epidemiological surveillance; blood, placenta and organ certification; prostate, cervical, colon and breast cancers; adult metabolics; and minimum-access neurosurgery.

We have 1562 laboratories/service centers in Cuba and 546 in other countries in the Americas, Africa and Asia. These include Brazil, Argentina, Mexico, Angola and China. 


\begin{tabular}{l|l}
\hline DIAGNOSTIC TOOL APPLICATION & $\begin{array}{c}\text { YEAR } \\
\text { INTRODUCED }\end{array}$ \\
\hline MATERNAL-CHILD PROGRAM & 1982 \\
\hline Congenital malformations (AFP) & 1992 \\
\hline Ectopic pregnancies, trophoblastic illnesses (HCG) & 1986 \\
\hline Congenital hypothyroidism (TSH) & 2000 \\
\hline Phenylketonuria (PKU) & 2005 \\
\hline Congenital adrenal hyperplasia & 2005 \\
\hline Biotinidase deficiency & 2005 \\
\hline Galactosemia & \\
\hline INFECTIOUS DISEASES & 1988 \\
\hline HIVIAIDS & 1988 \\
\hline Hepatitis B & 1993 \\
Hepatitis C & 2005 \\
\hline Hepatitis C (PCR) & 1990 \\
Dengue & 1990 \\
Leprosy & 1995 \\
Chagas & 1994 \\
\hline Tetanus & \\
\hline CHRONIC NON-COMIMUNICABLE DISEASES & 1987 \\
\hline Allergy (Total IgE) & 2008 \\
\hline Diabetes mellitus (SUMASENSOR) & 2004 \\
\hline Prostate cancer (PSA) & 2010 \\
\hline Microalbuminuria & 2012 \\
\hline Colorrectal cancer (SUMASOHF) & \\
\hline Source: Immunoassay Center, Havana & \\
\hline
\end{tabular}

We train both our domestic and international laboratory personnel, as part of post-marketing client services. In Cuba, this is accomplished at five regional centers that also provide technical assistance and evaluation to laboratories, which can be located in hospitals or stand-alone specialized centers.

MEDICC Review: How do you calculate the impact of SUMA technology on population health in Cuba? What are some of the most important results you can point to?

Niurka Carlos: I think one of our most important contributions has been in the area of postnatal testing. There are several diagnoses that need to be made at birth, to avoid development of serious conditions.

For example, congenital hypothyroidism leads to severe mental retardation. But, since we have tested for five of these conditions for years now (congenital hypothyroidism, phenylketonuria, congenital adrenal hyperplasia, biotinidase deficiency and galactosemia), through 2016 we have detected a total of 1039 newborns with one of these problems, and now we have among them university graduates. That is the result of massive screening, 9,559,807 tests in all.

In 2018, we expect to introduce testing for cystic fibrosis.
MEDICC Review: Some would say that represents a huge investment for a small number of infants.

Niurka Carlos: Yes, they might. But we believe it's not the statistic that counts, it's the child. You only need to think of the child as your own to realize how important these tests are. To you, that child is the most important in the world. And speaking of the world, over 126 million of these tests have been carried out abroad, diagnosing 7734 newborns in time.

In Cuba, we have also tested some 6.3 million pregnant women for HIV through 2016, a diagnosis that is followed by preventive measures to keep newborns from contracting the virus. As a result, WHO declared Cuba the world's first country to halt mother-tochild transmission of HIV.

It's also important for people to feel that their loved ones are protected from contracting infectious diseases through blood donations. In Cuba, we have a large national voluntary blood donation program. Over the last 28 years, our Center has been responsible for screening these donations for $\mathrm{HIV}$, hepatitis $\mathrm{B} / \mathrm{C}$ and tetanus, avoiding their transmission. So, people who need blood, whether they live in Havana or in the mountains of the Sierra Maestra, can count on an uncontaminated supply.

\section{MEDICC Review: How is the Center responding to new challenges?}

Niurka Carlos: In addition to improving our line of productswhich right now means converting semiautomated, multipart systems into a single automated system-we must continue to respond to the needs of the changing epidemiological picture in Cuba with new strategies and products, and to diversify our markets abroad.

If you look at the main causes of death in Cuba, you'll find cancer and heart/cerebrovascular diseases at the top of the list, accounting

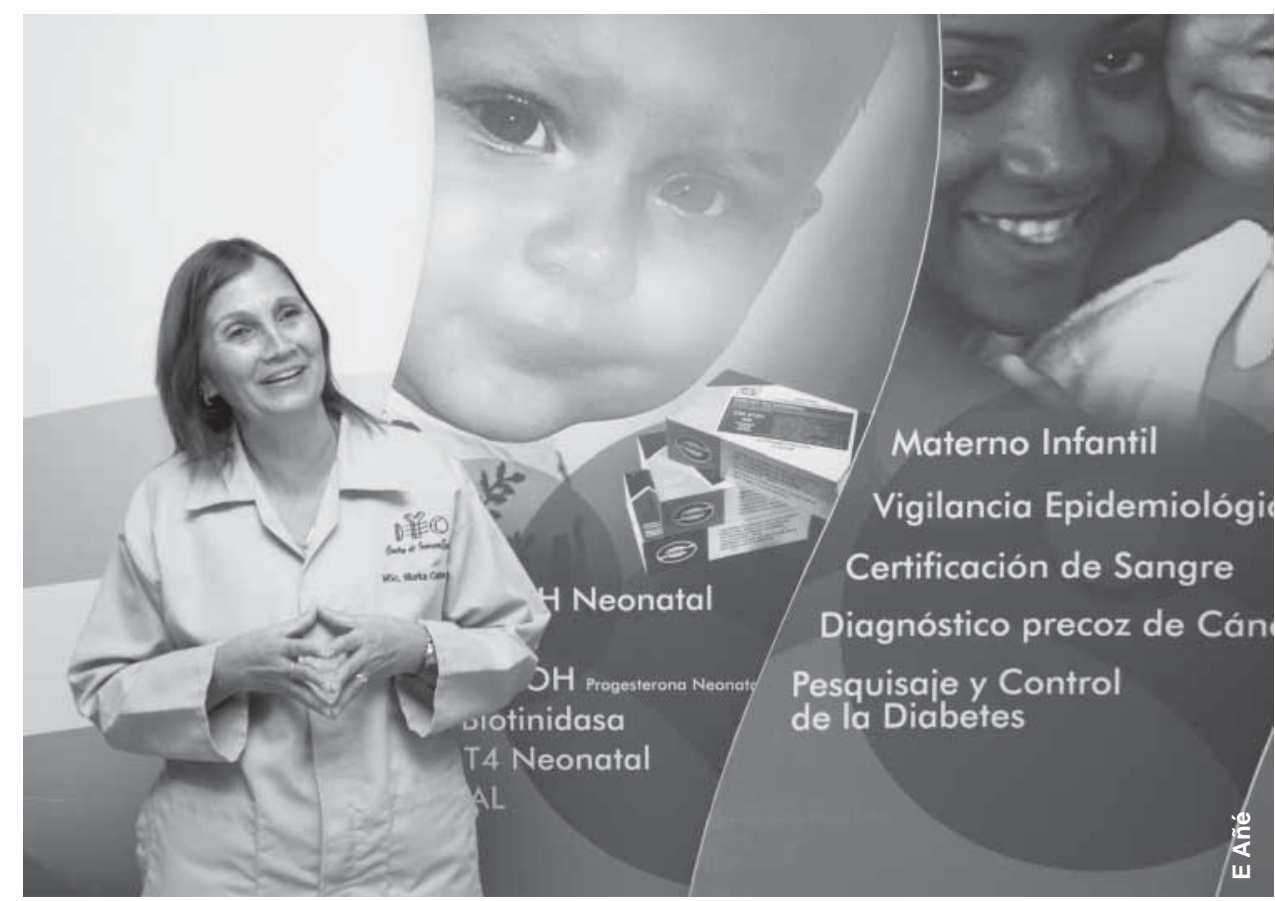


for $58 \%$ of all deaths. And if you consider that nearly $20 \%$ of our population is already at least 60 years old, then we have to foresee that these and related chronic conditions will only increase in the future. For example, diabetes mellitus is already the eighth cause of death in Cuba. If we can control diabetes, then we can stop progression to other chronic conditions. So, the challenge is not only to develop the technologies, but also to figure out how they can reach all the people who need them. In that respect, we have also introduced our glucometer (SUMASENSOR) into the health system as a whole. The inexpensive glucometer is universally available, for the first time enabling diabetic patients to accurately measure their glucose levels at home.

We have an integrated, universal health system whose foundation is strong primary care, including family doctors and nurses as well as community polyclinics. Now, in each of Cuba's 168 municipalities, we have a Specialized Center for Comprehensive Active Screening, which adds to primary care diagnostic effectiveness by ensuring technology at that level-closer to patients-for early diagnosis. This includes screening for chronic conditions such as diabetes, various cancers and chronic kidney disease, the latter through our test for microalbuminuria.

I think the prostate screening program through PSA is particularly interesting. Widespread screening with PSA was questioned in many quarters for a long time, but it has proved extremely effective in Cuba, where men are almost "allergic" to rectal exams. We began to introduce PSA into the national health system in 2012, and as a result, the pattern of the disease has been reversed. For example, in our study in Santiago, where physicians were diagnosing some $70 \%$ of prostate cancers in stages 3 and 4 , we found after incorporation of PSA, $70.8 \%$ are being caught in stages 1 and 2. This can make the difference between life and death. And you have to consider that prostate cancer is the second leading cause of cancer deaths in men in Cuba.

\section{MEDICC Review: You mentioned new strategies and pro- ducts. Can you be more specific?}

Niurka Carlos: Among our priorities, we're increasing our collaboration with different centers in BioCubaFarma as well as other institutions. For example, in terms of diagnostics, as you know the Molecular Immunology Center has developed the CIMAvax-EGF therapeutic vaccine for non-small cell lung cancer. Researchers are finding that the greater the presence of epidermal growth factor (EGF) in patients, the greater the response to this new therapy. So our technology (UMELISA EGF) is being applied to determine EGF levels in new patients, as a predictor of effectiveness.
We are also working with the Genetic Engineering and Biotechnology Center to better evaluate results of treatment with their medication for diabetic foot ulcers, Heberprot-P. Our contribution sounds like a simple one-a frame to ensure consistent positioning of the foot for followup photography (FrameHeber SUMA) - but if you think about it, it's essential that photos of the lesion are taken with the foot always in exactly the same position. Otherwise, it would be impossible to say whether a lesion is reappearing where already treated, or you are seeing a new lesion in another spot.

Finally, in the same vein, we have developed a stereotactic frame for minimum-access neurosurgery for the Neurological Restoration Center, where holding the patient's head in place is critical, especially for cases involving Parkinson disease. You also have to remember that this surgery is performed on patients who are awake.

There are of course other products in our pipeline, including rapid tests for various infectious diseases, new software models and new lines of technology for neonatal screening.

MEDICC Review: How are your new products evaluated, and do they have to be registered in Cuba?

Niurka Carlos: Absolutely. They go through a very rigorous evaluation process through the Cuban regulatory agency, CECMED (Center for State Control of Medicines, Equipment and Medical Devices). This involves multiple phases of assessment, in order to clearly demonstrate both the quality and safety of our products.

Every technology we introduce must also be registered in Cuba before we are permitted to export, and then the importing country registers it once again according to their own standards.

Unfortunately, because of the US embargo, we cannot export our technologies to the United States. But we believe they are especially important for low- and middle-income countries, since the prices are right and the products are flexible in terms of their use. That is, for example, they can be used in a centralized or decentralized way throughout a country, and health care services can use the fully automated version (as in Brazil or Mexico) or the partially automated versions of our diagnostics.

In any case, and in every nation, the use of these technologies for population health depends first and foremost-as I said earlieron the political will of the nation's policymakers, to ensure the broadest possible coverage and access. - $1 /$ - 